\title{
Energy Efficient Communication Protocol for Chain-based Clustered WSNs
}

\author{
Krishan Kumar ${ }^{1}$, Deepti Gupta ${ }^{2}$ and Nirmal Kaur ${ }^{3}$ \\ 1,2,3 UIET, Panjab University, Chandigarh, India \\ ${ }^{1}$ krishankumar1229@yahoo.in, ${ }^{2}$ deepti_gupta49@yahoo.co.in, \\ 3nirmaljul19@gmail.com
}

\begin{abstract}
Routing in Wireless Sensor networks greatly effects lifetime of the network as a major portion of the available energy is utilized for data transmission among nodes, and these nodes are very constrained in terms of their battery power, and it is not feasible to replace the battery over time. So specialized protocols are needed for data transmission in WSNs to develop such routing strategies that minimize the power consumption to enhance the overall lifetime of WSNs, but at the same time maintain the throughput of the network. Chain-based routing protocols try to achieve this by forming a chain of sensor nodes wherein each node pass on the data to its closest neighbor while the cluster based protocol divide the whole network into small clusters and then each node transmit the data to its respective cluster head. The protocol proposed in this paper utilizes both clustering and chaining strategies, and also the coordinator nodes are used to get an optimal path for data transmission that enhances the overall lifetime of the WSN. In the beginning, clusters of all the nodes are formed and then within each cluster, a chain is formed. Along with it, coordinator nodes are used for inter-cluster communication. These nodes receive the data from the lower level cluster which is transmitted to the respective chain head from where it is again transmitted to the upper cluster. With the help of simulation of the proposed protocol, it is observed that the routing strategy of the protocol outperforms Chain Based Cluster Cooperative Protocol (CBCCP) [9] and Power-Efficient Gathering in Sensor Information Systems (PEGASIS) [4] protocols in terms of the overall lifetime of the network.
\end{abstract}

Keywords: Wireless Sensor Networks (WSNs), Base Station (BS), Chain head, Coordinator Node

\section{Introduction}

Wireless Sensor Networks, commonly abbreviated as WSNs, refers to a special network in which the network devices are denoted as sensor nodes which can sense their surrounding environment and can communicate wirelessly with one another and to the outside network. The sensor nodes in case of WSNs can be categorized as the source node and sink node or base station. The source nodes sense the data from their deployed environment and then transmit it to the sink node, from where this data can be used locally or can be transmitted to other networks through the gateway. The source nodes can be stationary or move, homogeneous or heterogeneous, location-aware or location unaware and are resource constrained. With the advancement in the electrical components, the size, as well as the cost associated with the sensor nodes, has been reduced which make it possible to deploy a greater number of sensor nodes at a particular location to sense and transmit the gathered information to the desired location.

Received (April 10, 2018), Review Result (July 24, 2018), Accepted (August 22, 2018) 
A sensor node mainly consists of a sensing element, a controller, a transceiver, a memory unit and a power unit or battery. The sensor element is the sensor attached to the node that captures data from its deployed environment. It can measure any change in the physical property of the environment such as pressure, temperature, humidity and so on. The controller is the main component of the sensor node that performs tasks, processes the data captured by the sensor and controls the working of all the components in the sensor node. The transceiver component is responsible for the communication of data, both to and fro, in the node. The memory component can be used for storage of data in the node. The power unit or battery provides energy to the circuit of the sensor node to perform all the operations.

In case of Wireless Sensor and Actuator Networks, there is an additional component present called actuator. This component is responsible to perform some action on the environment. So, the flow of information is reversed in the case of an actuator, i.e. from the sink node to the actuator node. This enables WSANs to control the surrounding environment.

WSNs have certain properties which make them different from other networks which are discussed below:

1. WSNs are self-organizing networks.

2. WSNs are resource constrained networks, as there is the limited power that is associated with each sensor node.

3. Unlike other networks, in WSNs the node density is quite high.

4. In WSNs, the data that is transmitted by the nodes is small in size as compared to other networks.

5. The network topology of WSNs is highly dynamic.

6. The sensor nodes in WSNs have low complexity and low cost as compared to nodes of other networks.

7. In WSNs, due to higher node density, there is a higher degree of redundancy than other networks.

8. The flow of data is usually from multiple points to a single point.

The application of WSNs includes various areas such as environment monitoring, military surveillance, agricultural monitoring, healthcare, office and home automation, animal tracking and various industrial applications. WSNs act as a basic component for the Internet of Things (IoT) applications.

Due to the difference between WSNs and other networks, the routing protocols that exist for other networks are not suitable for WSNs. Moreover, the main goal of routing strategies in WSNs is an efficient use of the constrained energy associated with the network as it is not possible to recharge or replace the battery over time and functioning of the network need to be maintained for a desired amount of time. So separate routing protocols are needed for WSNs, some of which have been proposed and some are emerging as a result of the research being carried out for routing in WSNs.

The routing protocols for WSNs on the basis of their structure or topology can be classified into three main categories: Flat routing protocols, Hierarchical routing protocols, and Location-based protocols.

In flat routing protocols, all the sensor nodes have the same function and are provided with the same resources. In case of hierarchical routing protocols, some nodes are used for different operations than others which form a hierarchy of nodes. The location-based protocols are those in which the geographic information of the sensor nodes is used for making routing decisions. 
The proposed protocol is a hierarchical routing protocol that incorporates features from chain-based as well as clustering strategy.

In chain-based routing protocols, a chain of all the nodes in the network is formed and then a chain head is selected from the chain as in Power-Efficient Gathering in Sensor Information Systems (PEGASIS) [4]. Each node sends the sensed data to its nearest neighbor, where the neighbor node fuses its own data with the received data and transmits it to the next nearest neighbor. This procedure is repeated until the data from all the nodes reach the chain head from where it is sent to the base station. Chain-based protocols greatly prolong the lifetime of the network as the communication distance between the nodes is reduced.

In cluster-based routing protocols, the whole network is partitioned into various regions or clusters and a head node called the Cluster head $(\mathrm{CH})$ is selected from each cluster as in Low Energy Adaptive Clustering Protocol (LEACH) [1]. Each node in the network transmit the sensed data to its respective $\mathrm{CH}$. CHs from each cluster aggregates the data collected from all the nodes and transmit it to the sink node or base station.

\section{Related Works}

Various hierarchical routing protocols for WSNs have been proposed by different authors to cater the need of efficient use of energy in WSNs. Low Energy Adaptive Clustering Protocol (LEACH) [1] is a cluster based routing protocol wherein the whole network is partitioned into small clusters and a cluster head $(\mathrm{CH})$ is selected from each cluster. Each node in the network transmits the sensed data to its respective $\mathrm{CH}$, from where it is transmitted to the sink node or base station. The selection of $\mathrm{CH}$ is performed in each round and is rotated among all the nodes of a cluster. LEACH greatly improves the overall lifetime of the network as compared to the direct transmission.

LEACH-Centralized (LEACH-C) [2] is an extension of basic LEACH in which the base station defines the number of clusters that are to be formed so that the load of the network can be evenly distributed among different clusters.

Stable Election Protocol (SEP) [3] is also a cluster based protocol in which a heterogeneous network is formed. Some of the nodes in the network are advanced nodes. These nodes are allocated more resources as compared to the other normal nodes. The clustering is done in the same way as that of LEACH. The heterogeneity helps to increase the overall lifetime of the network.

Power-Efficient Gathering in Sensor Information Systems (PEGASIS) [4] is a chain based routing protocol in which a greedy chain of the nodes is formed. The chain-building starts from the node that is at a maximum distance from the base station. After the chain is formed, a random node from the chain is selected as Chain head. Each node in the network transmits the sensed data to its closest neighbor node where it is fused with the neighbor node's data and transmitted to the next closest node. This process continues until the data from all nodes reach to the chain head from where it is sent to the base station. The chain head node is changed after each round and is allocated on the basis of a nmodr factor, where $r$ is the round number and $n$ is the number of nodes. This protocol is able to achieve a lifetime of up to $300 \%$ more than LEACH.

Chain Cluster based Mixed routing (CCM) [5] is a chain-cluster based protocol that divides the whole network into a number of strips. For each strip, a chain is formed and chain head is selected. Then a cluster of all the chain heads is formed. From this cluster, a random node is selected as a cluster head. Data from all the chain heads is transmitted to this cluster head that sends the collected data to the base station. The role of the chain head, as well as cluster head, is changed after each round to manage the load balancing as is done in previous protocols.

Energy Efficient Chain Based routing (EECB) [6] is another chain based protocol wherein the selection of a node as a chain head is based on its distance from the base 
station and its remaining energy. Along with it, a distance threshold value for chain formation is used to reduce long links formed in the chain.

PEGASIS algorithm based on Double Cluster Heads (PDCH) [7] is another chain based protocol in which hierarchy of multiple chains is formed with two cluster heads in each chain, one from the main chain and other from the branch generated due to distance threshold value used for chain formation. The main chain head receives data from all the nodes in the chain through chaining while the secondary chain head transmits collected data to the next chain's head node.

Intersection Based Coverage Algorithm (IBCA) [8] is also chain based algorithm which aims to increase the lifetime of the network by finding redundant nodes in the network which can be put into a sleep state and the chain is formed for the rest nodes as done in PEGASIS.

In Chain Based Cluster Cooperative Protocol (CBCCP) [9], both clustering and chaining strategies are utilized. In the beginning, rectangular clusters of all the nodes are formed and then, Cluster head for each cluster is selected. This protocol assigns varied number nodes in each cluster, except lowest level cluster, as coordinator nodes to receive data from lower level clusters. Within each cluster, all the nodes transmit their data to their respective cluster head. The data from the cluster head is transmitted to the upperlevel cluster's coordinator node which is again transmitted to the next upper-level coordinator node until it reaches the topmost level from where it is transmitted to the base station. The number of coordinator nodes in a cluster is equal to the total number of clusters below it. This leads to the formation of a chain structure for inter-cluster communication.

Hybrid PEGASIS with Sink Mobility (HPSM) [10] is also a chain-cluster based routing protocol wherein the sink node or the base station is mobile. Sink node movement follows a predefined trajectory. The network is divided into various sub-areas and the chain is formed for each sub-area. The sink node moves from one region to another collecting data from the visited sub area in its path.

The study of various cluster and chain based protocols lead to the idea of the development of the proposed protocol that tries to utilize the advantages of both strategies to enhance network lifetime.

\section{Network Model}

The network model used for the WSNs in the proposed method has the following properties:

1. The network is homogeneous that means all the nodes in the network are provided with equal resources.

2. The network is static and there is no mobility in the nodes after deployment.

3. All the nodes in the sensor field are aware of their location.

4. The links in the network are symmetric.

5. There is a fixed amount of energy provided to each node which cannot be recharged.

6. In a particular round, all the nodes are assumed to contain some sensed data which they have to transmit over the network.

The energy model for evaluation of energy consumed by the nodes for data communication in every round is as follows:

To transmit k-bits of a data packet over some distance d, the energy consumed by a node is evaluated as:

$$
\operatorname{ETX}(\mathrm{k}, \mathrm{d})=\mathrm{E}_{\text {elec }} * \mathrm{k}+\operatorname{EMP} * \mathrm{k} * \mathrm{~d}^{2}, \text { for } \mathrm{d}<\mathrm{d}_{0}
$$




$$
\operatorname{ETX}(k, d)=E_{\text {elec }} * \mathrm{k}+\text { EFS } * \mathrm{k} * \mathrm{~d}^{4} \text {, for } \mathrm{d}>\mathrm{d}_{0}
$$

Where ETX represents the energy consumed by a node for the data transmission, Eelec is the energy consumed for transmitter operation, EMP and EFS are the amplification factors. For transmission over a distance less than a threshold value $\left(\mathrm{d}_{0}\right)$, EMP factor is used, and for distance larger than the threshold value $\left(\mathrm{d}_{0}\right)$, EFS factor is used. The threshold $\mathrm{d}_{0}$ is evaluated as:

$$
d_{0}=\sqrt{E F S / E M P}
$$

While receiving k-bit data the energy consumed is calculated as:

$$
\operatorname{ERX}(\mathrm{k})=\mathrm{E}_{\text {elec }} * \mathrm{k}
$$

For aggregating or fusing k-bit data packets the energy consumed is calculated as:

$$
\operatorname{EDA}(\mathrm{k})=\mathrm{E}_{\text {elec }} * \mathrm{k}
$$

\section{Proposed Protocol}

The protocol proposed in this paper, Energy Efficient Communication Protocol for Chain-based Clustered WSN (EECPCC), take advantages of clustering as well as chaining strategies to develop a novel technique that enhances the lifetime of the network. The main goal is to minimize the communication distance among the nodes in the network as most of the available energy is utilized for data transmission which is dependent on the communication distance. To achieve this, cluster-chains are formed which minimize the distance among the nodes within a cluster and use of the coordinator nodes help to reduce the inter-cluster communication distance. This prolongs the lifetime of the WSN. This protocol is developed for those regions where the base station has to be kept away from the sensor field.

The process of the proposed protocol follows two phases: a Setup phase and Steady phase. In the first phase, the network is initialized and the routing path is established among the nodes while in the second phase the data transmission takes place through the established route. These phases are discussed as follow:

\subsection{Setup Phase}

This is the first phase in which the path or route among the sensor nodes is formed in the network. An optimal path is determined so that the data that has to be transmitted to the base station has to travel minimum distance which in turn increases energy efficiency. This phase is completed in the following steps:

\subsubsection{Cluster Formation}

This is the first step of the setup phase. After all the nodes are deployed in the sensor field, the entire region is divided into small rectangular clusters on the basis of $\mathrm{x}$ and $\mathrm{y}$ coordinates. These clusters follow a hierarchical structure such that one cluster is above another. For the $100 \times 100 \mathrm{~m}^{2}$ network, 5 rectangular clusters are formed, each having a width of $20 \mathrm{~m}$. The subregion $(0-100) \times(0-20) \mathrm{m}$ is the first level cluster, $(0-100) \times(20-40) \mathrm{m}$ is the second level cluster and so on, up to $(0-$ $100) \times(80-100) m$ which is the last level (5th) cluster.

\subsubsection{Chain Formation}

After clustering has been done, all the nodes are aware of their respective clusters on the basis of their location. Now the next step in the proposed protocol is to form a chain for each cluster. Chain formation in the proposed protocol also follows a greedy approach which is similar to PEGASIS [4]. The chain-building in each 
cluster starts from the node that is farthest from the base station. The next node in the chain is one that is closest to the previous node. This process continues until a chain of all the nodes present in the cluster is formed.

\subsubsection{Selection of the Chain head and the Coordinator node}

After formation of cluster-chains, a random node from each cluster-chain is selected as Chain head. In each cluster chain, all the nodes transmit their sensed data to the selected chain head through chaining which is discussed later in steady phase.

Along with chain head, a coordinator node is also selected in each cluster-chain, except for the lowest level. The role of the coordinator node is to act as a link between two clusters. The chain head from the lower level cluster transmits the collected data to the coordinator node of the upper level. The selection of the coordinator node of a particular level cluster is done on the basis of its distance from the chain head of the lower level chain head. The node which is at a minimum distance from the chain head of the lower level is selected as the coordinator node.

The role of the chain head is shifted among all the nodes in a chain and is changed after every round so that the load is shared among all the nodes. As chain head is re-elected in every round, the coordinator node also changes automatically in every round as it is dependent on the distance from the chain head of lower level.

\subsection{Steady Phase}

After the completion of the setup phase, the next step is to transmit the data among the nodes based on the route that is established. The transmission of data in each chain starts from the end nodes of the chain. These nodes send their data to the closest node (next node in the chain) where this data gets fused with the next node's own data and is forwarded to the next neighbor. This process continues until the data from all the nodes in a cluster-chain is received by the chain head. The chain head of a particular level cluster-chain transmits the collected data to the coordinator node of the upper-level cluster-chain selected in the setup phase.

After receiving data from lower level chain head, the coordinator node sends it to its respective chain head which again transmits the collected data to its upper-level coordinator node. This process continues until all the data is reached at the topmost level. The chain head of the highest level cluster transmits the collected data of the network to the base station.

When any node in the chain dies due to exhaustion of energy or some failure in the node, only that chain needs to be re-established.

In Figure 1, a WSN of area 100x100 and 100 nodes is shown in which 5 clusterchains are formed. The sink node is present far away from the sensor field. Interchain links are formed with the help of coordinator nodes, shown with dashed line. The chain head of the highest level cluster-chain transmits the data to the sink node located at $(50,300)$. 


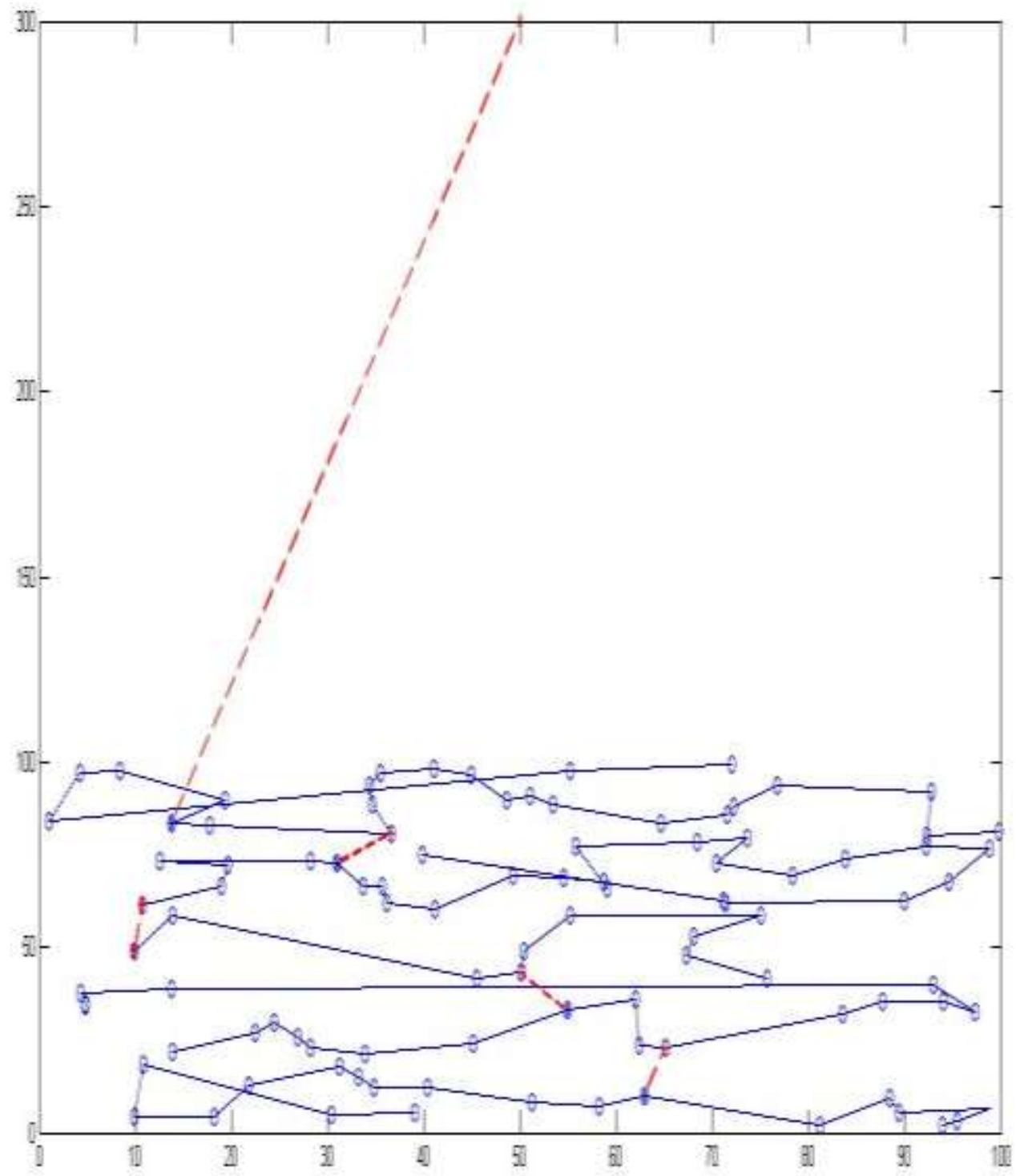

Figure 1. WSN having 100 Nodes in 100x100 Area

\subsection{Working of the Proposed Protocol}

For $\mathrm{n}$ number of nodes in 100x100 area and rmax rounds with the base station or sink node at $(50,300)$, the proposed protocol is implemented in the following steps:

Firstly, the random deployment of $n$ number of nodes in the sensing area takes place. These nodes are initialized with the same amount of initial energy, $E_{0}$. All the nodes are location aware and based on their coordinate values 5 clusters are formed, each of area size $100 \times 20 \mathrm{~m}$.

After the cluster formation, nodes within each cluster form a greedy chain. For each cluster, the node that is present at a maximum distance from the sink node is selected as start_node. The formation of the chain starts from the start_node. The next node in the chain is the one that is at a minimum distance from the start_node. This new node is selected as next start_node. This is repeated until all the nodes in the cluster are traversed. The resultant is a greedy chain such that every node within a cluster is connected to the closest neighbor node. 
The next step is the selection of the chain head and the coordinator node. From each cluster-chain, a random node is selected as the chain head. Along with it, a coordinator node is also selected for all the cluster-chains except the lowest level cluster. The coordinator node for a cluster-chain is that node which is at a minimum distance from the cluster head of its lower level cluster-chain. To balance the load, the role of chain head is changed after each round. As coordinator node is dependent on its distance from the chain head, it is also changed in each round with the change in the chain head.

The next step is the data transmission. For every round, each node has some sensed data to be transmitted to the base station. The transmission of data starts from the farthest cluster with respect to the sink node. Two types of transmissions take place in the proposed protocol. First is the intra-cluster transmission of data and the second is the inter-cluster transmission of data. In the intra-cluster data transmission, the data is transmitted among the nodes within a cluster-chain so that the sensed data from all the nodes can reach the respective chain head. The transmission for each chain starts from the end nodes within a chain. The end nodes transmit their sensed data to the neighbor node in the chain towards chain head. This node fuses its own data with the received data and transmits it to the next node in the chain. This process continues until data from all the nodes reach to the chain head. Now the inter-cluster data transmission takes place in which data from one cluster is transmitted to the other cluster so that it can be sent to the sink node. The chain head from the cluster-chain transmits the collected data to the coordinator node of the upper level where through intra-cluster transmission this data reaches to the chain head. This cluster head again transmits the collected data to the next level's coordinator node. This is repeated until data of the whole network reaches the chain head of the topmost chain. From the uppermost chain head, the collected data is transmitted to the base station.

For each round, the energy consumed by each node is calculated and the remaining energy is updated based on the energy model discussed in the previous section. When a node depletes all of its available energy then it becomes a dead node. Whenever a node dies in a chain in a particular round, the chain of the remaining alive nodes is re-established in the next round. Also if any fault occurs in any of the chains or all nodes are dead in any chain, then that chain can be omitted and routing occurs among the rest of the chains.

Figure 2 depicts this working of the proposed protocol in the form of a flowchart which is shown below: 


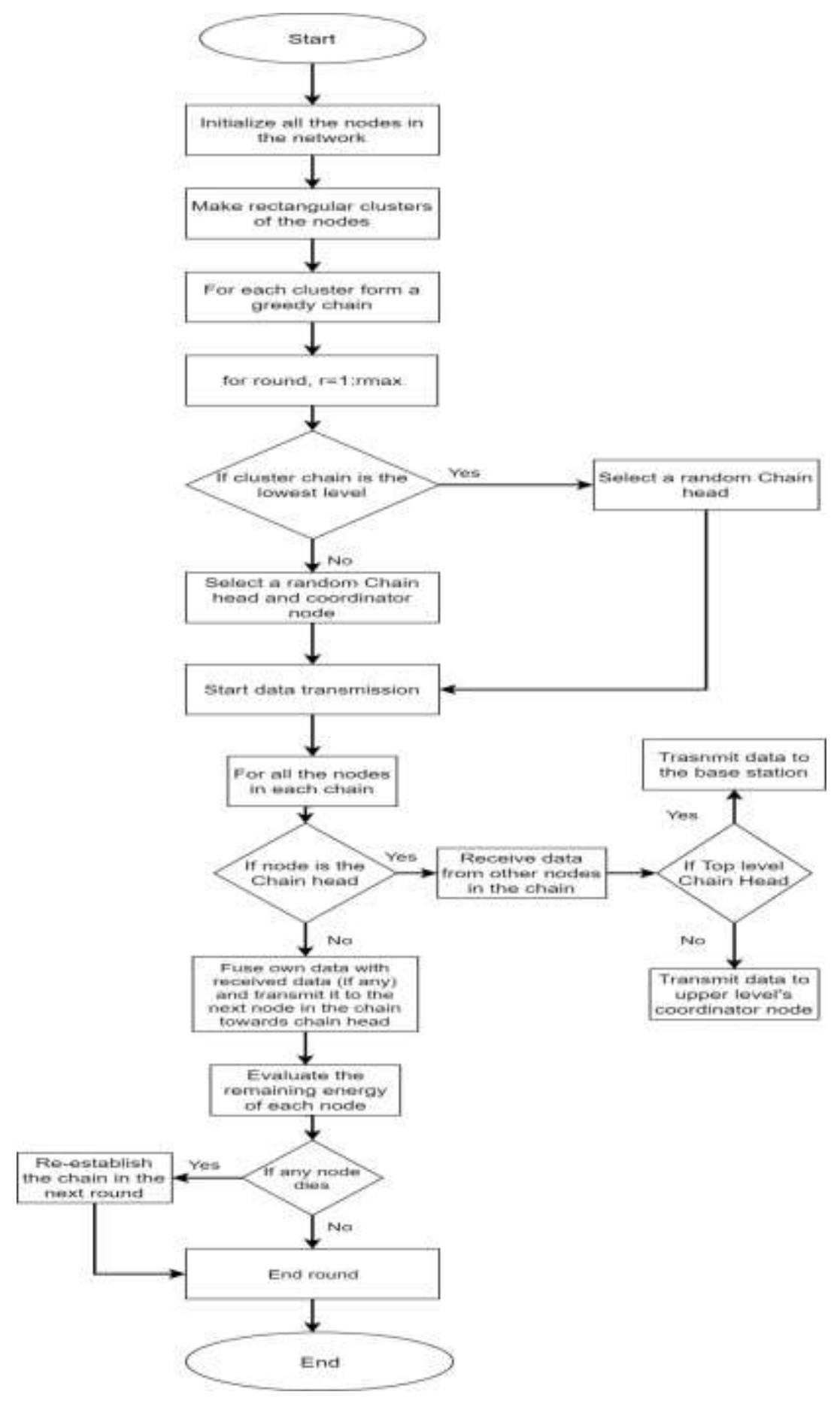

Figure 2. Flowchart of the Proposed Protocol

\section{Simulations}

The proposed protocol is implemented in Matlab to simulate the consumption of energy by the nodes in the deployed environment. For analyzing the network, the metrics used are: Number of dead nodes, Number of alive nodes and Remaining energy of the network. In the simulation, 100 nodes are randomly distributed over $100 \times 100$ cross-section area, with the sink node at $(50,300)$. The message size is taken as 4000 bits. Each node in the network has an initial energy of 0.5 Joules. 
Table 1. Simulation Parameters

\begin{tabular}{ll}
\hline Parameters & Values \\
Network Size & $(100 \times 100) \mathrm{m}^{2}$ \\
Number of nodes & 100 \\
Sink node location & $(50,300)$ \\
Initial energy & $0.5 \mathrm{~J}$ \\
Packet Size $(\mathrm{k})$ & $4000 \mathrm{bits}$ \\
E $_{\text {elec }}$ & $50 \times 10^{-9} \mathrm{~J} / \mathrm{bit}$ \\
EMP & $0.0013 \times 10^{-12} \mathrm{~J} / \mathrm{bit} / \mathrm{m}^{4}$ \\
EFS & $10 \times 10^{-12} \mathrm{~J} / \mathrm{bit} / \mathrm{m}^{2}$ \\
EDA & $5 \times 10^{-9} \mathrm{~J} / \mathrm{bit} / \mathrm{message}$ \\
\hline
\end{tabular}

\section{Performance Evaluation and Discussion}

For the simulated network area of $100 \times 100,5$ cluster-chains are formed. The proposed protocol, EECPCC, has been evaluated for transmission of 4000 bits of the data packet to the sink node located at $(50,300)$ and is compared with PEGASIS [4] and $\mathrm{CBCCP}[9]$ protocols. The parameters for the evaluation are Number of alive nodes, Number of dead nodes and Remaining energy of the network.

In Figure 3, a graph of dead nodes with respect to the number of rounds has been plotted for the EECPCC protocol, CBCCP protocol, and PEGASIS protocol.

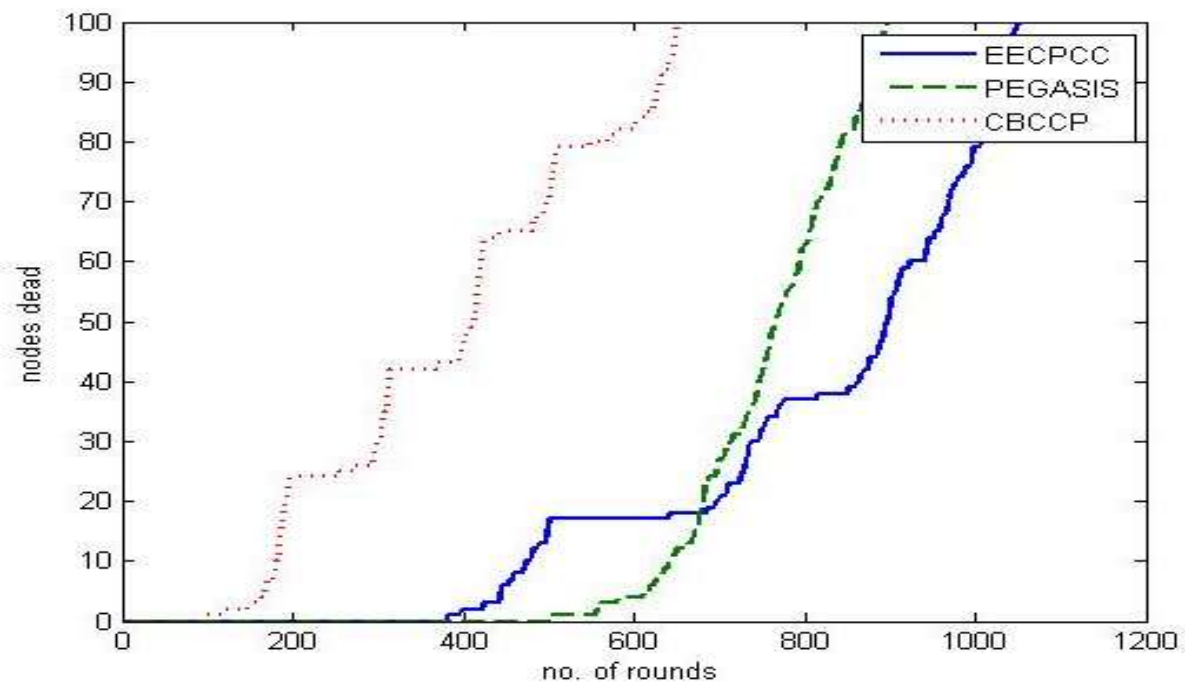

Figure 3. Comparison based on Dead Nodes

It is clear from the graph in Figure 3 that the nodes in CBCCP start dying much earlier than the proposed protocol. For PEGASIS, initially the node die slowly but later there is a sharp increase in the dead nodes and all the nodes die earlier than the proposed protocol.

In Figure 4, a graph of alive nodes with respect to the number of rounds has been plotted for the proposed EECPCC protocol, CBCCP protocol, and PEGASIS protocol. The graph depicts that the number of alive nodes for CBCCP in each round reduces faster than the proposed protocol. In PEGASIS initially the number of alive 
nodes in each round is more but later they reduce sharply and become lesser than that of the proposed protocol.

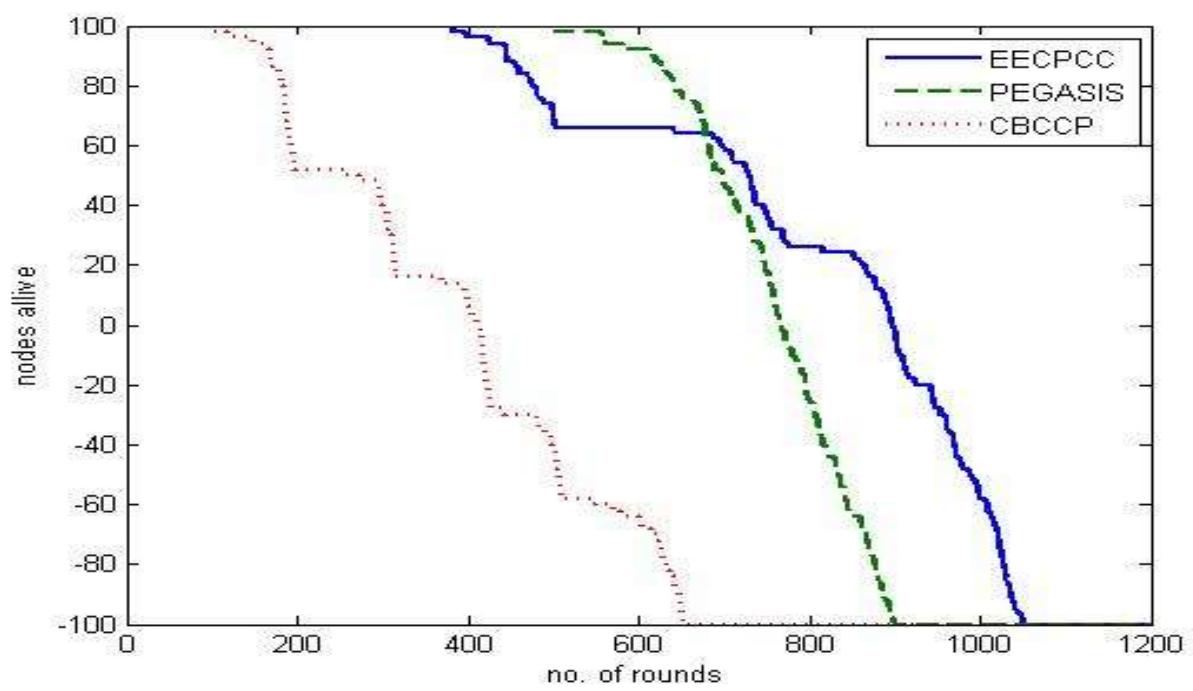

Figure 4. Comparison based on Alive Nodes

In Figure 5, a graph of the total energy remaining in the network with respect to the number of rounds has been plotted for the proposed EECPCC protocol, CBCCP protocol, and PEGASIS protocol.

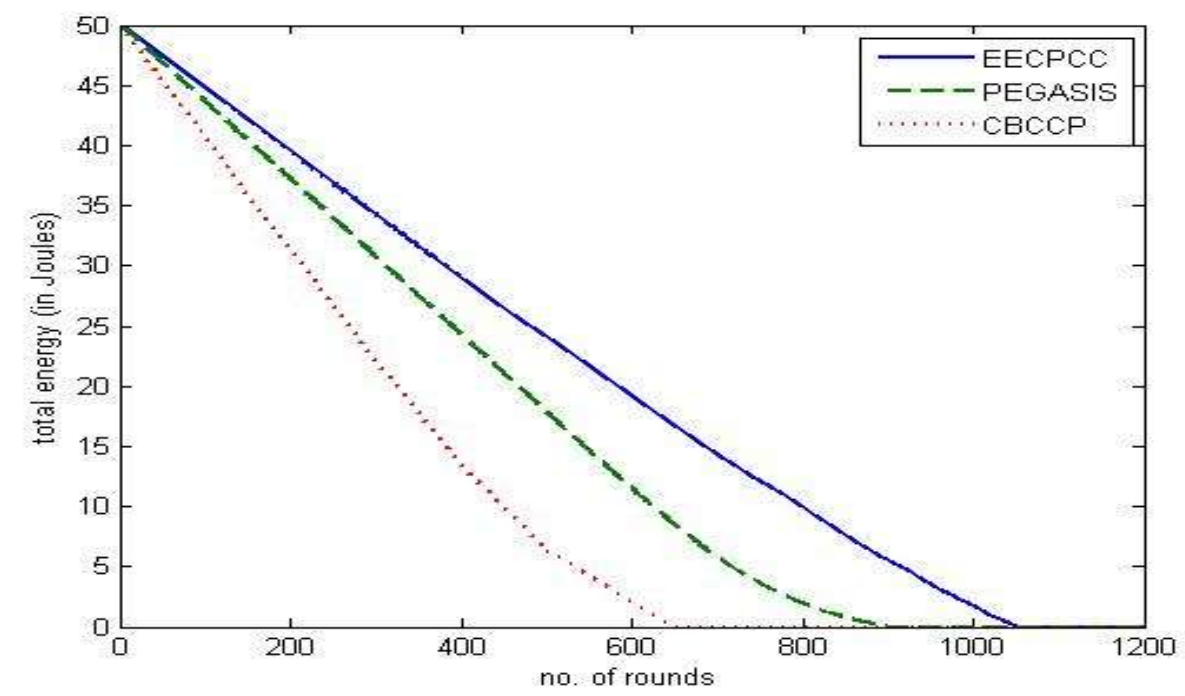

Figure 5. Comparison based on Total Energy Remaining in the Network

The total energy in Figure 5 is the sum of the energies of all the alive nodes in the network. The curves indicate that after each round, the remaining energy in case of the proposed protocol is greater than the PEGASIS and CBCCP protocols.

Based on results obtained from the simulation, it is evident that the proposed protocol provides better energy efficiency than PEGASIS and CBCCP, and increases the lifetime of the sensor network. The last node dies after 1050 rounds in the proposed protocol as against 898 in PEGASIS and 652 in CBCCP. 
In PEGASIS, where there is only a single chain, the fault or error in any intermediate node affect the routing process of the whole network, but in the proposed protocol there are multiple cluster-chains. So if there is some fault in one chain that chain can be ignored and the routing can take place among the rest of the cluster-chains which ensure connectivity and stability of the network. Also due to cluster chain formation, the long links which were there in PEGASIS also get reduced in the proposed protocol.

In $\mathrm{CBCCP}$, the intra-cluster communication is performed through coordination among nodes which is similar to cluster communication and chaining takes place for inter-cluster communication with varied number of coordinator nodes in each cluster without considering distance from the cluster head, while in the proposed protocol chaining is implemented for both intra-cluster as well as inter-cluster communication and distance from the chain head is considered for selecting the coordinator node. This minimizes the communication distance among the nodes and prolongs the lifetime of the network.

\section{Conclusion}

The new protocol presented in this paper achieves better energy efficiency while transmitting data to the base station that is present at a distant location from the sensor field. The balance of load is achieved by rotating the role of chain head, and minimum communication distance among nodes is achieved by forming cluster-chains and selecting coordinator nodes. This greatly reduces the energy consumed by the sensor nodes and improves the lifetime of the network. The network is scalable and new nodes can be easily attached to the network without affecting the performance of the proposed protocol. Also, using multiple chains ensures stability in the network. The use of sleep and wake schedule and redundancy control can further prolong the lifetime of the network which can be assessed in future work. This protocol fulfills the need of those areas where sensed data need to be communicated to a distant base station in an energy efficient way like in case of military surveillance.

\section{References}

[1] W. R. Heinzelman, A. Chandrakasan and H. Balakrishnan, "Energy-efficient communication protocol for wireless microsensor networks", Proceedings of the 33rd annual Hawaii international conference on System Sciences, (2000) January 4.

[2] W. B. Heinzelman, A. P. Chandrakasan and H. Balakrishnan, "An application-specific protocol architecture for wireless microsensor networks", IEEE Transactions on wireless communications, vol. 1, no. 4, (2002) pp. 660-670.

[3] G. Smaragdakis, I. Matta and A. Bestavros, "SEP: A stable election protocol for clustered heterogeneous wireless sensor networks", Boston University Computer Science Department, (2004).

[4] S. Lindsey and C. S. Raghavendra, "PEGASIS: Power-efficient gathering in sensor information systems", Proceedings of IEEE Aerospace conference, (2002).

[5] F. Tang, I. You, S. Guo, M. Guo and Y. Ma, "A chain-cluster based routing algorithm for wireless sensor networks", Journal of Intelligent Manufacturing, vol. 35, no. 4, (2012), pp. 1305-1313.

[6] Y. Yu and Y. Song, "An energy-efficient chain-based routing protocol in wireless sensor network", Proceedings of Computer Application and System Modeling (ICCASM), (2010) October 22-24.

[7] W. Linping, B. Wu, C. Zhen and W. Zufeng, "Improved algorithm of PEGASIS protocol introducing double cluster heads in wireless sensor network", Proceedings of Computer, Mechatronics, Control and Electronic Engineering (CMCE), (2010) August 24-26.

[8] Y. L. Chen, Y. C. Lin and N. C. Wang, "An intersection-based coverage algorithm for PEGASIS architecture in Wireless Sensor Networks", Proceedings of Machine Learning and Cybernetics (ICMLC), 2012 International Conference, (2012) July 15.

[9] S. Rani, J. Malhotra and R. Talwar, "Energy efficient chain based cooperative routing protocol for WSN", Applied Soft Computing, vol. 35, (2015) October 31, pp. 386-397.

[10] A. Semwal, A. Chamoli, K. Singh and A. Bhatt, "To increase network lifespan of wireless sensor network by hybrid pegasis with sink mobility", Proceedings of Inventive Systems and Control (ICISC), 2017 International Conference, (2017) January 19. 